\title{
A Novel Highly Stereoselective Synthesis of 2,3-Disubstituted 3H-Quinazoline-4-one Derivatives
}

Paul Zhichkin* ${ }^{\dagger}$, Edward Kesicki ${ }^{\dagger}$, Jennifer Treiberg ${ }^{\ddagger}$, Lisa Bourdon ${ }^{\dagger}$, Matthew Ronsheim $^{\dagger}$, Hua Chee Ooi ${ }^{\ddagger}$, Stephen White ${ }^{\ddagger}$, Angela Judkins ${ }^{\ddagger}$, and David Fairfax ${ }^{\dagger}$.

Albany Molecular Research, Inc., 21 Corporate Circle P.O. Box 15098, Albany, New York, 12212 and ICOS Corp., $2202120^{\text {th }}$ Ave SE, Bothell, WA, 98021.

pavelz@albmolecular.com

$\underline{\text { Table of Contents }}$

General Information and Experimental Procedures

Page S2-S14

References

Page $\mathrm{S} 14$

Spectra

Page S15-S 
General: Unless otherwise noted, reagents and solvents were used as received from commercial suppliers. Proton and carbon nuclear magnetic resonance spectra were obtained on a Bruker AC 300, Bruker AV 300 or Bruker DPX 300 spectrometer at 300 $\mathrm{MHz}$ for proton and $75 \mathrm{MHz}$ for carbon, or on a Varian Mercury VX 400 spectrometer at $400 \mathrm{MHz}$ for proton and $100 \mathrm{MHz}$ for carbon, or on a Bruker AMX 500 spectrometer at $500 \mathrm{MHz}$ for proton and $125 \mathrm{MHz}$ for carbon. Spectra are given in ppm $(\delta)$ and coupling constants, $J$, are reported in Hertz. Tetramethylsilane was used as an internal standard for proton and carbon spectra. High resolution mass spectra were obtained on a TOF mass spectrometer using electrospray ionization at the Center for Functional Genomics, University at Albany (Albany, NY). Elemental analyses were performed by Quantitative Technologies, Inc. (Whitehouse, NJ).

\section{Synthesis of 2-amino-6-methyl- $N$-o-tolylbenzamide (1).}<smiles>Cc1ccccc1NC(=O)c1c(C)cccc1N</smiles>

6-Methylanthranilic acid $(10.0 \mathrm{~g}, 66.2 \mathrm{mmol})$ was heated at reflux with a mixture of thionyl chloride $(20 \mathrm{~mL}, 32.6 \mathrm{~g}, 274 \mathrm{mmol})$ and toluene $(100 \mathrm{~mL})$. After $1 \mathrm{~h}$ the reaction was concentrated in vacuo, and the resulting residue was dissolved in THF $(100 \mathrm{~mL}) . O-$ Toluidine (21 mL, $21.0 \mathrm{~g}, 196 \mathrm{mmol})$ was added dropwise maintaining the internal temperature at $5-10{ }^{\circ} \mathrm{C}$. The reaction mixture was then heated at reflux for $18 \mathrm{~h}$. After this time the reaction mixture was cooled to ambient temperature, quenched with $10 \%$ aqueous $\mathrm{K}_{2} \mathrm{CO}_{3}(100 \mathrm{~mL})$ and stirred vigorously for $15 \mathrm{~min}$. The organic layer was separated, dried over $\mathrm{Na}_{2} \mathrm{SO}_{4}$ and evaporated under reduced pressure. The resulting solid was purified by flash chromatography on silica gel eluting with a gradient from 10:90 to 70:30 ethyl acetate/hexanes to afford $\mathbf{1}(14.5 \mathrm{~g}, 91 \%)$ as a light off-white solid: $\mathrm{mp} 110$ $112{ }^{\circ} \mathrm{C} ;{ }^{1} \mathrm{H}$ NMR $\left(300 \mathrm{MHz}, \mathrm{CDCl}_{3}\right) \delta 7.94(\mathrm{~d}, J=7.9 \mathrm{~Hz}, 1 \mathrm{H}), 7.37$ (br s, 1H), 7.27$7.20(\mathrm{~m}, 2 \mathrm{H}), 7.13-7.04(\mathrm{~m}, 2 \mathrm{H}), 6.61(\mathrm{~d}, J=1.5 \mathrm{~Hz}, 1 \mathrm{H}), 6.55(\mathrm{~d}, J=8.1 \mathrm{~Hz}, 1 \mathrm{H}), 4.14$ (br s, 2H), 2.43 (s, 3H), 2.28 (s, 3H); ${ }^{13} \mathrm{C}$ NMR (75 MHz, $\left.\mathrm{CDCl}_{3}\right) \delta 167.69,144.9,135.4$, 135.3, 130.7, 130.3, 129.4, 126.8, 125.5, 123.2, 122.9, 120.4, 114.0, 20.3, 18.1. Anal. Calcd for $\mathrm{C}_{15} \mathrm{H}_{16} \mathrm{~N}_{2} \mathrm{O}: \mathrm{C}, 74.97 ; \mathrm{H}, 6.71 ; \mathrm{N}, 11.66$. Found: C, 74.72; H, 6.80; N, 11.53.

\section{Synthesis of tert-butyl (S)-1-[3-methyl-2-(o-tolylcarbamoyl)phenylamino]-1- oxopropan-2-ylcarbamate (7).}<smiles>Cc1ccccc1NC(=O)c1c(C)cccc1NC(=O)[C@H](C)NC(=O)OC(C)(C)C</smiles>

A solution of 1 (2.40 g, $10.0 \mathrm{mmol})$, Boc-L-alanine (2.06 g, $10.5 \mathrm{mmol})$ and 1-hydroxy-7azabenzotriazole $(1.36 \mathrm{~g}, 10.0 \mathrm{mmol})$ in DMF $(16 \mathrm{~mL})$ was treated with 1-ethyl-3-[3dimethylamino)propyl]carbodiimide hydrochloride $(2.50 \mathrm{~g}, 13.0 \mathrm{mmol})$, and the reaction was stirred for $18 \mathrm{~h}$ at room temperature. After this time the solvent was evaporated in vacuo $(0.3 \mathrm{mmHg})$ at $40{ }^{\circ} \mathrm{C}$ on a rotary evaporator. The resulting residue was suspended 
in a mixture of water $(100 \mathrm{~mL})$ and $\mathrm{CH}_{2} \mathrm{Cl}_{2}(20 \mathrm{~mL})$, and the suspension was filtered. The filter cake was washed with $\mathrm{CH}_{2} \mathrm{Cl}_{2}(10 \mathrm{~mL})$ followed by $10 \%$ aqueous $\mathrm{K}_{2} \mathrm{CO}_{3}(10$ $\mathrm{mL})$ and water $(2 \times 10 \mathrm{~mL})$ and dried in vacuo to afford $7(2.72 \mathrm{~g}, 66 \%)$ as a white solid: mp 190-192 ${ }^{\circ} \mathrm{C} ;{ }^{1} \mathrm{H}$ NMR (300 MHz, DMSO-d $\left.d_{6}\right) \delta 9.86(\mathrm{~s}, 1 \mathrm{H}), 9.26(\mathrm{~s}, 1 \mathrm{H}), 7.73(\mathrm{~d}, J=$ $8.7 \mathrm{~Hz}, 1 \mathrm{H}), 7.58(\mathrm{~d}, J=7.5 \mathrm{~Hz}, 1 \mathrm{H}), 7.32(\mathrm{t}, J=7.9 \mathrm{~Hz}, 1 \mathrm{H}), 7.18-7.08(\mathrm{~m}, 5 \mathrm{H}), 4.11$ (br t, $J=6.9 \mathrm{~Hz}, 1 \mathrm{H}), 2.43(\mathrm{~s}, 3 \mathrm{H}), 2.28(\mathrm{~s}, 3 \mathrm{H}), 1.33(\mathrm{~s}, 9 \mathrm{H}), 1.23(\mathrm{~d}, J=7.0 \mathrm{~Hz}, 3 \mathrm{H})$; ${ }^{13} \mathrm{C}$ NMR $\left(75 \mathrm{MHz}, \mathrm{DMSO}-d_{6}\right) \delta 172.0,165.9,155.3,135.8,135.0,134.7,132.5,130.5$, 130.3, 128.9, 126.4, 126.1, 125.8, 125.7, 120.7, 78.3, 50.7, 28.1, 19.8, 18.2, 17.7. HRMS Calcd for $\mathrm{C}_{23} \mathrm{H}_{29} \mathrm{~N}_{3} \mathrm{O}_{4}+\mathrm{H}: 412.2236$. Found: 412.2239 .

\section{Synthesis of tert-butyl (S)-1-(5-methyl-4-(o-tolylimino)-4H-benzo[d][1,3]oxazin-2- yl)ethylcarbamate (8).}

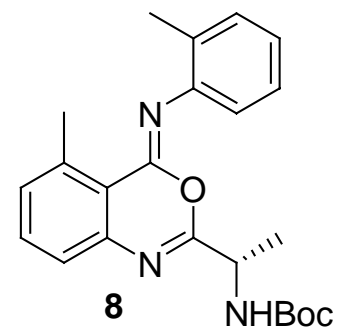

Bromine $(125 \mu \mathrm{L}, 390 \mathrm{mg}, 2.43 \mathrm{mmol})$ was added to a solution of $\mathrm{PPh}_{3}(630 \mathrm{mg}, 2.40$ $\mathrm{mmol})$ in $\mathrm{CH}_{2} \mathrm{Cl}_{2}(12 \mathrm{~mL})$. The solution was stirred for $30 \mathrm{~min}$, and triethylamine $(0.84$ $\mathrm{mL}, 0.613 \mathrm{~g}, 6.07 \mathrm{mmol})$ was added followed by 6 (822 mg, $2.00 \mathrm{mmol})$. Boiling of the solvent was observed as a result exothermic reaction. After the exotherm subsided, the reaction was heated at reflux for a further $1.5 \mathrm{~h}$. After this time the solution was cooled to room temperature and extracted with saturated aqueous $\mathrm{NaHCO}_{3}(2 \times 25 \mathrm{~mL})$. The organic layer was separated, dried over $\mathrm{Na}_{2} \mathrm{SO}_{4}$ and concentrated in vacuo. The resulting residue was purified by flash chromatography on silica gel eluting with $50 \%$ solution of ethyl acetate in hexanes containing $1 \%$ triethylamine to give $8(786 \mathrm{mg}, 26 \%)$ as a brown wax: $\mathrm{mp} 47-50{ }^{\circ} \mathrm{C} ;{ }^{1} \mathrm{H}$ NMR $\left(300 \mathrm{MHz}, \mathrm{CDCl}_{3}\right) \delta 7.43(\mathrm{t}, J=7.7 \mathrm{~Hz}, 1 \mathrm{H}), 7.29-7.21(\mathrm{~m}$, $3 \mathrm{H}), 7.17(\mathrm{t}, J=8.0 \mathrm{~Hz}, 1 \mathrm{H}), 7.02(\mathrm{td}, J=7.4,1.2 \mathrm{~Hz}, 1 \mathrm{H}), 6.92(\mathrm{dd}, J=8.9,1.0 \mathrm{~Hz}$, $1 \mathrm{H}), 5.22$ (br s, 1H), 4.39 (br t, $J=6.5 \mathrm{~Hz}, 1 \mathrm{H}), 2.85(\mathrm{~s}, 3 \mathrm{H}), 2.22(\mathrm{~s}, 3 \mathrm{H}), 1.44(\mathrm{~s}, 9 \mathrm{H})$, $1.32(\mathrm{~d}, J=7.0 \mathrm{~Hz}) ;{ }^{13} \mathrm{C} \mathrm{NMR}\left(75 \mathrm{MHz}, \mathrm{CDCl}_{3}\right) \delta 160.0,154.9,144.7,144.5,143.5$, 140.6, 132.5, 131.4, 130.3, 129.2, 126.1, 124.5, 123.8, 120.3, 117.6, 79.8, 48.5, 28.4, 24.2, 19.6, 18.4. HRMS Calcd for $\mathrm{C}_{23} \mathrm{H}_{27} \mathrm{~N}_{3} \mathrm{O}_{3}+\mathrm{H}$ : 394.2130. Found: 394.2130.

\section{Conversion of 8 into tert-butyl (S)-1-(5-methyl-4-oxo-3-o-tolyl-3,4- dihydroquinazolin-2-yl)ethylcarbamate (3a).}<smiles>Cc1ccccc1-n1c([C@@H](C)NC(=O)OCc2ccccc2)nc2cccc(C)c2c1=O</smiles>

A solution of 8 (194 mg, $0.494 \mathrm{mmol})$ in pyrrolidine $(1.0 \mathrm{~mL})$ was stirred overnight at room temperature. After this time the solvent was evaporated; the resulting residue was dissolved in isopropyl acetate $(20 \mathrm{~mL})$, and the solvent was evaporated again. A $1 \%$ solution of acetic acid in acetonitrile $(10 \mathrm{~mL})$ was added, and the mixture was refluxed for $30 \mathrm{~min}$. The reaction was then concentrated to a residue, which was purified by flash chromatography on silica gel eluting with $20 \%$ ethyl acetate in hexanes to afford 3a (104 $\mathrm{mg}, 94 \%$ ) with $96 \%$ ee. For the general procedure of ee determination and characterization of 3a see the general procedure below. 


\section{Synthesis of 2-methyl-6-nitro- $\mathrm{N}$-o-tolylbenzamide (9a).}<smiles>Cc1ccccc1NC(=O)c1c(C)cccc1[N+](=O)[O-]</smiles>

A mixture of 2-methyl-6-nitrobenzoic acid $(2.00 \mathrm{~g}, 11.0 \mathrm{mmol})$, toluene $(15 \mathrm{~mL})$ and thionyl chloride $(3.0 \mathrm{~mL}, 4.89 \mathrm{~g}, 41.0 \mathrm{mmol})$ was heated at reflux for $2 \mathrm{~h}$. The reaction was then concentrated at $45^{\circ} \mathrm{C}$ under reduced pressure, and THF (30 mL) was added to the resulting residue, followed by $N, N$-diisopropylethylamine $(5.0 \mathrm{~mL}, 3.7 \mathrm{~g}, 28.7 \mathrm{mmol})$ and $o$-toluidine $(1.2 \mathrm{~mL}, 1.2 \mathrm{~g}, 11.2 \mathrm{mmol})$. After the exothermic reaction subsided, the reaction mixture was stirred at ambient temperature for a further $2 \mathrm{~h}$. The solution was treated with $10 \%$ aqueous $\mathrm{K}_{2} \mathrm{CO}_{3}(30 \mathrm{~mL})$; the THF layer was separated, washed with brine and evaporated in vacuo. The solid residue was triturated with 2:1 ethyl acetate/hexanes $(20 \mathrm{~mL})$ and dried under vacuum to afford $9 \mathbf{a}(2.66 \mathrm{~g}, 89 \%)$ as a light brown solid. The obtained material contained $10 \%$ by weight of $N, N$ diisopropylethylamine hydrochloride. A small sample was recrystallized from toluene and characterized: mp $121-122{ }^{\circ} \mathrm{C} ;{ }^{1} \mathrm{H}$ NMR (500 MHz, DMSO- $\left.d_{6}\right) \delta 10.02(\mathrm{~s}, 1 \mathrm{H}), 8.05$ $(\mathrm{d}, J=8.2 \mathrm{~Hz}, 1 \mathrm{H}) ; 7.76(\mathrm{~d}, J=7.6 \mathrm{~Hz}, 1 \mathrm{H}), 7.62(\mathrm{t}, J=7.9 \mathrm{~Hz}, 1 \mathrm{H}), 7.50(\mathrm{~d}, J=7.8 \mathrm{~Hz}$, 1H), $7.25(\mathrm{~m}, 2 \mathrm{H}), 7.15$ (t, $J=7.4,1 \mathrm{H}), 2.49$ (s, 3H, overlaps with DMSO signal), 2.27 $(\mathrm{s}, 3 \mathrm{H}) ;{ }^{13} \mathrm{C}$ NMR $\left(125 \mathrm{MHz}, \mathrm{DMSO}-d_{6}\right) \delta 164.2,145.9,137.2,136.2,135.6,132.8$, 132.3, 130.4, 129.5, 126.0, 125.8, 125.4, 121.8, 18.7, 17.8. Anal. Calcd for $\mathrm{C}_{15} \mathrm{H}_{14} \mathrm{~N}_{2} \mathrm{O}_{3}$ : C, 66.66; H, 5.22; N, 10.36. Found: C, 66.36; H, 5.13; N, 10.26.

\section{Synthesis of 2-methyl-6-nitro- $N$-phenylbenzamide (9b).}<smiles>Cc1cccc([N+](=O)[O-])c1C(=O)Nc1ccccc1</smiles>

A mixture of 2-methyl-6-nitrobenzoic acid ( $8.00 \mathrm{~g}, 44.0 \mathrm{mmol})$, toluene (95 $\mathrm{mL})$ and thionyl chloride $(33.0 \mathrm{~mL}, 22.0 \mathrm{~g}, 185 \mathrm{mmol})$ was heated at reflux for $2 \mathrm{~h}$. The reaction was then concentrated at $45{ }^{\circ} \mathrm{C}$ under reduced pressure, and THF ( $\left.80 \mathrm{~mL}\right)$ was added to the resulting acid chloride. With cooling in an ice/water bath, $N, N$-diisopropylethylamine $(23 \mathrm{~mL}, 17.0 \mathrm{~g}, 132 \mathrm{mmol})$ and aniline $(4.0 \mathrm{~mL}, 4.1 \mathrm{~g}, 44.0 \mathrm{mmol})$ were added. After this addition was complete, the reaction mixture was stirred at ambient temperature overnight. The mixture was then concentrated under reduced pressure, and the residue was dissolved in $\mathrm{CH}_{2} \mathrm{Cl}_{2}(100 \mathrm{~mL})$. This solution was washed with $10 \%$ aqueous citric acid $(100 \mathrm{~mL})$, dried over $\mathrm{Na}_{2} \mathrm{SO}_{4}$ and concentrated in vacuo to afford $9 \mathbf{b}(11.3 \mathrm{~g}$, quantitative yield) as an off-white solid: mp 146-148 ${ }^{\circ} \mathrm{C} ;{ }^{1} \mathrm{H}$ NMR (300 MHz, DMSO- $d_{6}$ ) $\delta 10.59(\mathrm{~s}, 1 \mathrm{H}), 8.05(\mathrm{~d}, J=8.1 \mathrm{~Hz}, 1 \mathrm{H}), 7.77(\mathrm{~d}, J=7.6 \mathrm{~Hz}, 1 \mathrm{H}), 7.76-7.60(\mathrm{~m}, 3 \mathrm{H})$, $7.36(\mathrm{t}, J=7.7 \mathrm{~Hz}, 2 \mathrm{H}), 7.12(\mathrm{t}, J=7.4 \mathrm{~Hz}, 1 \mathrm{H}), 2.41(\mathrm{~s}, 3 \mathrm{H}) ;{ }^{13} \mathrm{C}$ NMR $(75 \mathrm{MHz}$, DMSO- $\left.d_{6}\right) \delta 164.3,146.2,139.2,137.6,136.7,133.0,130.1,129.2,124.3,122.2,120.0$, 19.0; Anal. Calcd for $\mathrm{C}_{14} \mathrm{H}_{12} \mathrm{~N}_{2} \mathrm{O}_{3}$ : C, 65.62; H, 4.72; N, 10.93. Found: C, 65.63; H, 4.43; $\mathrm{N}, 10.79$.

\section{Synthesis of $N$-(3,5-difluorophenyl)-2-methyl-6-nitrobenzamide (9c).}




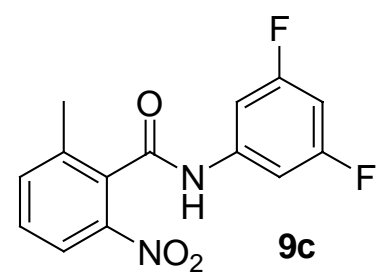

Following the procedure for the preparation of $9 \mathbf{b}$, the acid chloride prepared from 2methyl-6-nitrobenzoic acid (10.0 g, $55.0 \mathrm{mmol})$ was reacted with 3,5-difluoroaniline $(7.10 \mathrm{~g}, 55.0 \mathrm{mmol})$ in refluxing THF for $6 \mathrm{~h}$. Final purification by flash chromatography on silica gel, eluting with $25 \%$ ethyl acetate in hexanes, afforded $9 \mathrm{c}(9.28 \mathrm{~g}, 58 \%)$ as a brown solid: mp 158-160 ${ }^{\circ} \mathrm{C} ;{ }^{1} \mathrm{H}$ NMR (300 MHz, DMSO- $\left.d_{6}\right) \delta 10.99(\mathrm{~s}, 1 \mathrm{H}), 8.09$ (d, $J$ $=8.1 \mathrm{~Hz}, 1 \mathrm{H}), 7.79(\mathrm{~d}, J=7.6 \mathrm{~Hz}, 1 \mathrm{H}), 7.67$ (t, $J=8.0 \mathrm{~Hz}, 1 \mathrm{H}), 7.36$ (dd, $J=2.2,9.5 \mathrm{~Hz}$, $2 \mathrm{H}), 7.00(\mathrm{tt}, J=9.4,2.3 \mathrm{~Hz}, 1 \mathrm{H}), 2.40(\mathrm{~s}, 3 \mathrm{H}) ;{ }^{13} \mathrm{C}$ NMR $\left(75 \mathrm{MHz}, \mathrm{DMSO}-d_{6}\right) \delta 164.5$, $162.5(\mathrm{dd}, J=243.6,15.2 \mathrm{~Hz}), 145.6,141.1(\mathrm{t}, J=13.6 \mathrm{~Hz}), 137.3,136.6,131.9,130.1$, 121.9, $102.3(\mathrm{dd}, 19.3,9.9 \mathrm{~Hz}), 99.1(\mathrm{t}, J=26.1)$, 18.4. Anal. Calcd for $\mathrm{C}_{14} \mathrm{H}_{10} \mathrm{~F}_{2} \mathrm{~N}_{2} \mathrm{O}_{3}: \mathrm{C}$, 57.54; H, 3.45; N, 9.59. Found: C, 57.71; H, 3.48; N, 9.55.

\section{Synthesis of $N$-benzyl-2-nitrobenzamide $(9 \mathrm{~d}){ }^{1}$}<smiles>O=C(NCc1ccccc1)c1ccccc1[N+](=O)[O-]</smiles>

Benzylamine (13 mL, $12.7 \mathrm{~g}, 119 \mathrm{mmol})$ was added dropwise to a solution of 2nitrobenzoyl chloride $(10.0 \mathrm{~g})$ in $\mathrm{CH}_{2} \mathrm{Cl}_{2}(100 \mathrm{~mL})$, maintaining the internal temperature at $15-25{ }^{\circ} \mathrm{C}$. The reaction was then stirred at ambient temperature for $30 \mathrm{~min}$ and diluted with water $(100 \mathrm{~mL})$. The organic layer was separated, extracted with $10 \%$ aqueous citric acid $(100 \mathrm{~mL})$ followed by saturated aqueous $\mathrm{NaHCO}_{3}(100 \mathrm{~mL})$, dried over $\mathrm{Na}_{2} \mathrm{SO}_{4}$ and concentrated in vacuo. The resulting residue was dissolved in toluene (50 $\mathrm{mL})$ at $70{ }^{\circ} \mathrm{C}$, and this solution was then cooled to room temperature. Hexanes $(100 \mathrm{~mL})$ were added, and the suspension was cooled to $0{ }^{\circ} \mathrm{C}$ and filtered. The filter cake was dried in vacuo to afford 9d (11.5 g, 84\%) as a light brown solid: $\mathrm{mp} 121-122{ }^{\circ} \mathrm{C},{ }^{1} \mathrm{H}$ NMR (300 $\left.\mathrm{MHz} \mathrm{CDCl}_{3}\right) \delta 9.21(\mathrm{t}, J=5.7 \mathrm{~Hz}, 1 \mathrm{H}), 8.04(\mathrm{dd}, J=7.9,1.0 \mathrm{~Hz}, 1 \mathrm{H}), 7.80(\mathrm{td}, J=7.4$, $1.1 \mathrm{~Hz}, 1 \mathrm{H}), 7.72-7.64(\mathrm{~m}, 2 \mathrm{H}), 7.36(\mathrm{~m}, 4 \mathrm{H}), 7.27(\mathrm{~m}, 1 \mathrm{H}), 4.46(\mathrm{~d}, J=6.0 \mathrm{~Hz}, 2 \mathrm{H})$.

\section{Synthesis of 2-fluoro-6-nitro- $N$-phenylbenzamide (9f).}<smiles>O=C(Nc1ccccc1)c1c(F)cccc1[N+](=O)[O-]</smiles>

2-Fluoro-6-nitrobenzoic acid (100 g, $0.540 \mathrm{~mol}$ ) was suspended in a mixture of DMF (5 $\mathrm{mL})$ and $\mathrm{CH}_{2} \mathrm{Cl}_{2}(600 \mathrm{~mL})$. A $2 \mathrm{M}$ solution of oxalyl chloride in $\mathrm{CH}_{2} \mathrm{Cl}_{2}(410 \mathrm{~mL}, 0.820$ mol) was added dropwise over $30 \mathrm{~min}$. After $1 \mathrm{~h}$ an aliquot was removed and analyzed by ${ }^{1} \mathrm{H}$ NMR spectroscopy. The spectroscopy showed that complete conversion to the acid chloride had occurred. The reaction mixture was concentrated under reduced pressure to give an orange syrup, which contained a small amount of solid material. The acid chloride was used in the next reaction without additional purification.

A mixture of aniline (49 mL, $50.2 \mathrm{~g}, 0.540 \mathrm{~mol})$ and $\mathrm{NaHCO}_{3}(90.0 \mathrm{~g}, 1.08 \mathrm{mmol})$ with 1,4-dioxane $(250 \mathrm{~mL})$ and water $(250 \mathrm{~mL})$ was cooled to $6{ }^{\circ} \mathrm{C}$ in an ice bath. A solution of the acid chloride prepared above in 1,4-dioxane $(80 \mathrm{~mL})$ was added over $10 \mathrm{~min}$. 
During this addition the temperature reached a maximum of $27{ }^{\circ} \mathrm{C}$. The reaction mixture was stirred overnight and then quenched by addition of water $(1.2 \mathrm{~L})$. After stirring for an additional $15 \mathrm{~min}$, the solid was collected by filtration, washed with water and dried in a vacuum oven at $50{ }^{\circ} \mathrm{C}$ to give $9 f(139 \mathrm{~g}, 99 \%)$ as a beige solid: $\mathrm{mp} 163-165{ }^{\circ} \mathrm{C} ;{ }^{1} \mathrm{H}$ NMR (400 MHz, DMSO- $\left.d_{6}\right) \delta 10.88$ (br s, $\left.1 \mathrm{H}\right), 8.13(\mathrm{~d}, J=8.0 \mathrm{~Hz}, 1 \mathrm{H}), 7.81-7.88(\mathrm{~m}$, $1 \mathrm{H}), 7.79(\mathrm{dd}, J=8.2,5.8 \mathrm{~Hz}, 1 \mathrm{H}), 7.72(\mathrm{dd}, J=8.4,1.0 \mathrm{~Hz}, 2 \mathrm{H}), 7.41(\mathrm{t}, J=8.0 \mathrm{~Hz}$, $2 \mathrm{H}), 7.16(\mathrm{t}, J=7.4 \mathrm{~Hz}, 1 \mathrm{H}) ;{ }^{13} \mathrm{C}$ NMR $\left(100 \mathrm{MHz}, \mathrm{DMSO}-d_{6}\right) \delta 159.1,158.6(\mathrm{~d}, J=$ $249.5 \mathrm{~Hz}), 146.4(\mathrm{~d}, J=5.3 \mathrm{~Hz}), 138.6,131.8(\mathrm{~d}, J=9.2 \mathrm{~Hz}), 129.0,124.3,122.4(\mathrm{~d}, J=$ $22.1 \mathrm{~Hz}), 121.7(\mathrm{~d}, J=23.7 \mathrm{~Hz}), 120.6(\mathrm{~d}, J=3.1 \mathrm{~Hz}), 119.6$. Anal. Calcd for $\mathrm{C}_{13} \mathrm{H}_{9} \mathrm{FN}_{2} \mathrm{O}_{3}: \mathrm{C}, 60.00 ; \mathrm{H}, 3.49 ; \mathrm{N}, 10.77$. Found: C, 59.91; H, 3.14; N, 10.68.

\section{Synthesis of $N$-(2,6-difluorophenyl)-2-nitrobenzamide (9g).}<smiles>O=C(Nc1c(F)cccc1F)c1ccccc1[N+](=O)[O-]</smiles>

A solution of 2-nitrobenzoyl chloride (25.0 g, $135 \mathrm{mmol})$ in 1,4-dioxane (50 mL) was added dropwise to a solution of 2,6-difluoroaniline (36 mL, $43.5 \mathrm{~g}, 337 \mathrm{mmol}$ ) in 1,4dioxane $(350 \mathrm{~mL})$. The resulting yellow suspension was stirred at ambient temperature overnight. After this time the reaction was diluted with water $(400 \mathrm{~mL})$ and stirred for 30 min. The solids were then collected by filtration, washed with water and dried in vacuo to afford 9g (33.4 g, 97\%) as a white solid: $\mathrm{mp} 184-186{ }^{\circ} \mathrm{C} ;{ }^{1} \mathrm{H}$ NMR (400 MHz, DMSO$\left.d_{6}\right) \delta 10.53(\mathrm{~s}, 1 \mathrm{H}) ; 8.15(\mathrm{~d}, J=8.0 \mathrm{~Hz}, 1 \mathrm{H}), 7.89(\mathrm{dt}, J=7.5,1.0 \mathrm{~Hz}, 1 \mathrm{H}), 7.83-7.74(\mathrm{~m}$, $2 \mathrm{H}) ; 7.48-7.37(\mathrm{~m}, 1 \mathrm{H}), 7.22(\mathrm{t}, J=8.2 \mathrm{~Hz}, 2 \mathrm{H}) ;{ }^{13} \mathrm{C}$ NMR $\left(100 \mathrm{MHz}, \mathrm{DMSO}-d_{6}\right) \delta$ $164.4,157.8(\mathrm{dd}, J=249.9,5.0 \mathrm{~Hz}), 146.9,133.9,131.44,131.40,129.3,128.6(\mathrm{t}, J=9.5$ $\mathrm{Hz}), 124.3,113.7$ (t, $J=16.8 \mathrm{~Hz}), 112.0(\mathrm{dd}, J=17.9,5.0 \mathrm{~Hz})$. Anal. Calcd for $\mathrm{C}_{13} \mathrm{H}_{8} \mathrm{~F}_{2} \mathrm{~N}_{2} \mathrm{O}_{3}$ : C, 56.12; H, 2.90; N, 10.07; F, 13.66. Found: C, 56.22; H, 2.85; N, 10.07; $\mathrm{F}, 13.29$.

\section{Synthesis of $N$-(2,6-difluorophenyl)-2-methyl-6-nitrobenzamide (9h).}<smiles>Cc1cccc([N+](=O)[O-])c1C(=O)Nc1c(O)cccc1F</smiles>

Thionyl chloride $(121 \mathrm{~mL}, 1.66 \mathrm{~mol})$ was added dropwise to a solution of 2-nitro-6methyl benzoic acid (100 g, $552 \mathrm{mmol})$ in THF (1.2 L), and the reaction was stirred at ambient temperature for 18 hours. After this time the mixture was concentrated in vacuo, and the resulting acid chloride was dissolved in 1,4-dioxane $(250 \mathrm{~mL})$. This solution was added dropwise to a solution of 2,6-difluoroaniline $(178 \mathrm{~mL}, 214 \mathrm{~g}, 1.66 \mathrm{~mol})$ in 1,4dioxane $(750 \mathrm{~mL})$. The reaction was then stirred at ambient temperature overnight. Addition of water $(1.2 \mathrm{~L})$ resulted in a brown precipitate, which was collected by filtration, washed with water and dried in vacuo to afford $9 \mathrm{~h}(71.7 \mathrm{~g}, 45 \%)$ as a light brown solid: mp184-186 ${ }^{\circ} \mathrm{C} ;{ }^{1} \mathrm{H}$ NMR (400 MHz, DMSO- $\left.d_{6}\right) \delta 10.47$ (br s, $\left.1 \mathrm{H}\right), 7.37$ (br $\mathrm{s}, 1 \mathrm{H}), 8.06-8.04(\mathrm{~m}, 1 \mathrm{H}), 7.76-7.74(\mathrm{~m}, 1 \mathrm{H}), 7.62(\mathrm{t}, J=8.0 \mathrm{~Hz}, 1 \mathrm{H}), 7.44-7.37(\mathrm{~m}, 1 \mathrm{H})$, $7.21(\mathrm{t}, J=8.0 \mathrm{~Hz}, 2 \mathrm{H}), 2.50(\mathrm{~s}, 3 \mathrm{H}) ;{ }^{13} \mathrm{C} \mathrm{NMR}\left(100 \mathrm{MHz}, \mathrm{CDCl}_{3}\right) \delta 165.1,158.7(\mathrm{dd}, J$ $=250.3,5.3 \mathrm{~Hz}), 146.7,138.2,137.0,132.7,130.6,129.2(\mathrm{t}, J=9.6 \mathrm{~Hz}), 122.5,114.3(\mathrm{t}$, $J=17.2 \mathrm{~Hz}$ ), 112.7 (dd, $J=18.3,4.6 \mathrm{~Hz}$ ), 19.3. Anal. Calcd for $\mathrm{C}_{14} \mathrm{H}_{10} \mathrm{~F}_{2} \mathrm{~N}_{2} \mathrm{O}_{3}: \mathrm{C}, 57.54$; H, 3.45; N, 9.59. Found: C, 57.60; H, 3.08; N, 9.47. 
Synthesis of 2-methyl-6-nitro- $N$-o-tolylbenzimidoyl chloride (10a) and tert-butyl $(S)$ 1-(2-methyl-6-nitro- $N$-o-tolylbenzamido)-1-oxopropan-2-ylcarbamate (11a).<smiles>Cc1ccccc1/N=C(\Cl)c1c(C)cccc1[N+](=O)[O-]</smiles><smiles>Cc1ccccc1N(C(=O)c1c(C)cccc1[N+](=O)[O-])C(=O)[C@H](C)NC(C)(C)C</smiles>

A mixture of $9 \mathbf{a}(1.50 \mathrm{~g}, 5.55 \mathrm{mmol})$, toluene $(15 \mathrm{~mL})$, thionyl chloride $(3.0 \mathrm{~mL}, 4.86 \mathrm{~g}$, $40.8 \mathrm{mmol})$ and DMF $(20 \mu \mathrm{L})$ was refluxed for $2.5 \mathrm{~h}$. The resulting solution was concentrated under reduced pressure at $45{ }^{\circ} \mathrm{C}$ to afford crude $10 \mathrm{a}$ as a yellow air-sensitive oil: ${ }^{1} \mathrm{H}$ NMR $\left(300 \mathrm{MHz}, \mathrm{CDCl}_{3}\right) \delta 8.06(\mathrm{~d}, J=8.2 \mathrm{~Hz}, 1 \mathrm{H}), 7.62(\mathrm{~d}, J=7.4 \mathrm{~Hz}, 1 \mathrm{H}), 7.52$ $(\mathrm{t}, J=7.9 \mathrm{~Hz}, 1 \mathrm{H}), 7.29(\mathrm{~d}, J=7.4 \mathrm{~Hz}, 1 \mathrm{H}), 7.25$ (overlaps with $\mathrm{CHCl}_{3}$ signal, $1 \mathrm{H}$ ), 7.15 $(\mathrm{t}, J=7.4 \mathrm{~Hz}, 1 \mathrm{H}), 7.08(\mathrm{~d}, J=7.7 \mathrm{~Hz}, 1 \mathrm{H}), 2.62(\mathrm{~s}, 3 \mathrm{H}), 2.28(\mathrm{~s}, 3 \mathrm{H}) ;{ }^{13} \mathrm{C}$ NMR $(75$ $\left.\mathrm{MHz}, \mathrm{CDCl}_{3}\right) \delta 146.3,145.2,137.7,136.3,132.5,130.6,129.9,129.1,126.4,125.8$, $122.5,119.2,19.5,18.0$. This material was dissolved in $\mathrm{CH}_{2} \mathrm{Cl}_{2}(4.0 \mathrm{~mL})$ and added to a solution of Boc-L-alanine (873 mg, $4.26 \mathrm{mmol})$ and $N, N$-diisopropylethylamine $(1.5 \mathrm{~mL}$, $1.1 \mathrm{~g}, 8.5 \mathrm{mmol})$ in $\mathrm{CH}_{2} \mathrm{Cl}_{2}(6 \mathrm{~mL})$ at $0-5{ }^{\circ} \mathrm{C}$. The reaction mixture was stirred for $1 \mathrm{~h}$ at this temperature and then warmed to room temperature overnight. The resulting solution was washed with $10 \%$ aqueous citric acid $(2 \times 15 \mathrm{~mL})$ followed by saturated aqueous $\mathrm{NaHCO}_{3}(10 \mathrm{~mL})$, dried over sodium sulfate and concentrated in vacuo. Purification by flash chromatography on silica gel eluting with $20 \%$ ethyl acetate in hexanes afforded 11a $(1.80 \mathrm{~g}, 88 \%)$ as a light brown foam: $\mathrm{mp} 72-74{ }^{\circ} \mathrm{C}$; according to the ${ }^{1} \mathrm{H}$ and ${ }^{13} \mathrm{C}$ NMR spectra the obtained material is a mixture of several rotamers: ${ }^{1} \mathrm{H} \mathrm{NMR}(300 \mathrm{MHz}$, $\left.\mathrm{CDCl}_{3}\right) \delta 8.2-8.0(\mathrm{~m}, 1 \mathrm{H}), 7.8-7.2(\mathrm{~m}, 6 \mathrm{H}), 5.02(\mathrm{~d}, J=8.9 \mathrm{~Hz}, 0.2 \mathrm{H}), 4.9-4.7(\mathrm{~m}, 0.8 \mathrm{H})$, 4.21 (br s, 0.6H), 4.05 (br s, 0.4H, overlaps with ethyl acetate signal), 2.52 (s, 0.6H), 2.48 $(\mathrm{s}, 3.5 \mathrm{H}), 2.45(\mathrm{~s}, 1.5 \mathrm{H}), 2.38(\mathrm{~s}, 0.4 \mathrm{H}), 1.27(\mathrm{~s}, 9 \mathrm{H}), 1.18(\mathrm{~d}, J=6.9 \mathrm{~Hz}, 0.7 \mathrm{H}), 1.12(\mathrm{~d}, J$ $=6.9 \mathrm{~Hz}, 1.4 \mathrm{H}), 1.04(\mathrm{~d}, J=6.9 \mathrm{~Hz}, 0.3 \mathrm{H}), 0.99(\mathrm{~d}, J=6.6 \mathrm{~Hz}, 0.6 \mathrm{H}) ;{ }^{13} \mathrm{C} \mathrm{NMR}(75$ $\left.\mathrm{MHz}, \mathrm{CDCl}_{3}\right) \delta 176.3,175.7,175.6,168.3,168.0,167.8,155.4,154.8,154.4,145.2$, $144.9,144.0,138.6,137.1,136.5,136.4,136.3,135.0,134.1,131.8,130.1,130.0,129.9$, $129.5,129.2$, 128.8, 128.7, 128.6, 128.4, 127.6, 127.4, 127.1, 122.2, 121.9, 121.8, 79.9, 79.8, 49.5, 49.1, 28.1, 21.0, 19.9, 19.7, 19.2, 19.1, 18.9, 18.5, 18.3, 18.1, 17.7, 17.6, 16.4. Anal. Calcd for $\mathrm{C}_{23} \mathrm{H}_{27} \mathrm{~N}_{3} \mathrm{O}_{6}$ : C, 62.57; H, 6.16; N, 9.52. Found: C, 62.59; H, 6.15; N, 9.49 .

\section{Synthesis of tert-butyl (S)-1-(2-methyl-6-nitro- $N$-phenylbenzamido)-1-oxopropan-2- ylcarbamate (11b).}

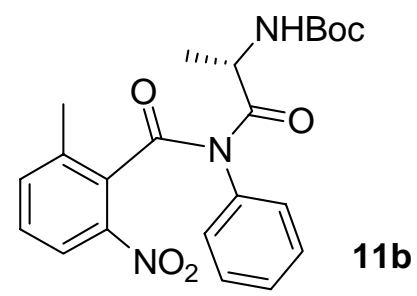

The procedure for the preparation of 11a has been followed, starting with $9 \mathrm{~b}(2.00 \mathrm{~g}$, $7.81 \mathrm{mmol})$ and Boc-L-alanine $(1.57 \mathrm{~g}, 8.28 \mathrm{mmol})$. Final purification by flash chromatography on silica gel eluting with a gradient from $100 \%$ hexanes to $50 \%$ ethyl acetate in hexanes, afforded $11 \mathbf{b}(2.39 \mathrm{~g}, 80 \%)$ as an off-white solid: $\mathrm{mp} 68-70{ }^{\circ} \mathrm{C} ;{ }^{1} \mathrm{H}$ NMR (300 MHz, $\left.\mathrm{CDCl}_{3}\right) \delta 8.09(\mathrm{~s}, 1 \mathrm{H}), 7.61-7.43(\mathrm{~m}, 8 \mathrm{H}), 4.87$ (br m, 1H), 4.22 (br s, $1 \mathrm{H}), 2.45(\mathrm{~s}, 3 \mathrm{H}), 1.38(\mathrm{~s}, 9 \mathrm{H}), 1.21-1.10$ (br m, 3H); ${ }^{13} \mathrm{C} \mathrm{NMR}\left(75 \mathrm{MHz}, \mathrm{CDCl}_{3}\right) \delta$ $175.7,168.5,155.2,154.6,144.7,144.0,136.3,136.0,135.1,134.8,130.0,130.7,129.2$, 
128.6, 121.7, 79.9, 49.4, 28.2, 18.9, 18.6, 17.2. Anal. Calcd for $\mathrm{C}_{22} \mathrm{H}_{25} \mathrm{~N}_{3} \mathrm{O}_{6}: \mathrm{C}, 61.82 ; \mathrm{H}$, 5.90; N, 9.83. Found: C, 61.81; H, 5.90; N, 9.77.

Synthesis of tert-butyl (S)-1-( $N$-(3,5-difluorophenyl)-2-methyl-6-nitrobenzamido)-1oxopropan-2-ylcarbamate (11c).

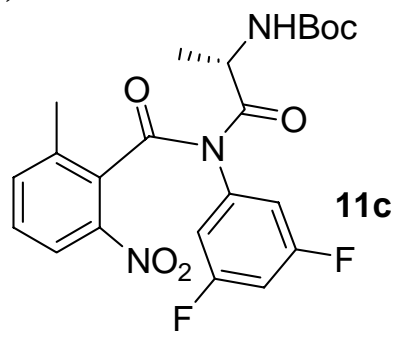

The procedure for the preparation of $11 \mathrm{a}$ has been followed, starting with $9 \mathbf{c}(6.35 \mathrm{~g}, 21.7$ $\mathrm{mmol})$ and Boc-L-alanine $(4.93 \mathrm{~g}, 26.0 \mathrm{mmol})$. Final purification by trituration with methanol afforded 11c $(6.35 \mathrm{~g}, 63 \%)$ as an off-white solid: $\mathrm{mp} 184-188{ }^{\circ} \mathrm{C} ;{ }^{1} \mathrm{H} \mathrm{NMR}$ $\left(500 \mathrm{MHz}, \mathrm{CDCl}_{3}\right) \delta 8.08$ (br d, $\left.J=5.6 \mathrm{~Hz}, 1 \mathrm{H}\right), 7.55$ (d, $\left.J=7.2 \mathrm{~Hz}, 1 \mathrm{H}\right), 7.45$ (br t, $J=$ 7.1 Hz, 1H), 7.30-6.90 (m, 3H), 5.00-4.65 (br m, 1H), 4.13 (br s, 1H), 2.45 (s, 3H), 1.38 (s, 9H), 1.25-1.00 (br m, 3H); $\left.{ }^{13} \mathrm{C} \mathrm{NMR} \mathrm{(125} \mathrm{MHz,} \mathrm{CDCl}_{3}\right) \delta 175.0,168.2,163.4$ (dd, $J=$ $252.3,14.9 \mathrm{~Hz}), 155.3,144.5,144.0,138.5,137.0,136.4,136.1,134.5,129.1,128.8$, 122.0, 121.8, 113.4 (d, $J=19.1 \mathrm{~Hz}), 105.8(\mathrm{t}, J=25.5 \mathrm{~Hz}), 80.2,49.4,28.2$, 18.9, 17.0. Anal. Calcd for $\mathrm{C}_{23} \mathrm{H}_{27} \mathrm{~N}_{3} \mathrm{O}_{6}$ : C, 57.02; H, 5.00; N, 9.07. Found: C, 57.25; H, 4.70; N, 9.04 .

Synthesis of $N$-benzyl-2-nitrobenzimidoyl chloride (10d) and tert-butyl $(S)-1-(N$ benzyl-2-nitrobenzamido)-1-oxopropan-2-ylcarbamate (11d).<smiles>O=[N+]([O-])c1ccccc1/C(Cl)=N/Cc1ccccc1</smiles><smiles>C[C@H](NC(=O)OCc1ccccc1)C(=O)N(Cc1ccccc1)C(=O)c1ccccc1[N+](=O)[O-]</smiles>

Following the procedure for the preparation of 10a, 9d (2.56 g, $10.0 \mathrm{mmol})$ was converted into crude 10d (2.87 g, >100\%) as a yellow air-sensitive oil: ${ }^{1} \mathrm{H}$ NMR (300 $\left.\mathrm{MHz} \mathrm{CDCl}_{3}\right) \delta 7.97(\mathrm{~d}, J=7.9 \mathrm{~Hz}, 1 \mathrm{H}), 7.66(\mathrm{~m}, 2 \mathrm{H}), 7.59(\mathrm{~m}, 1 \mathrm{H}), 7.58-7.25(\mathrm{~m}, 5 \mathrm{H})$, $4.89(\mathrm{~s}, 2 \mathrm{H}) ;{ }^{13} \mathrm{C} \mathrm{NMR}\left(125 \mathrm{MHz}, \mathrm{CDCl}_{3}\right) \delta 147.1,138.6,137.1,133.2,130.9,130.3$, 128.6, 127.9, 127.4, 124.4, 58.1. Crude 10d (550 mg, $2.00 \mathrm{mmol})$ was then reacted with Boc-L-alanine (400 mg, $2.12 \mathrm{mmol}$ ) following the procedure for the preparation of 11a. Purification by flash chromatography on silica gel eluting with $33 \%$ ethyl acetate in hexanes afforded 11d $\left(611 \mathrm{mg}, 74 \%\right.$ over two steps) as a light brown foam: $\mathrm{mp} 55-58{ }^{\circ} \mathrm{C}$; ${ }^{1} \mathrm{H}$ NMR $\left(500 \mathrm{MHz}, \mathrm{CDCl}_{3}\right) \delta 8.21(\mathrm{~d}, J=8.8 \mathrm{~Hz}, 1 \mathrm{H}), 7.56(\mathrm{~m}, 2 \mathrm{H}), 7.28(\mathrm{~m}, 3 \mathrm{H}), 7.18$ (br m, 3H), 5.07 (br m, 4H), 1.43 (s, 9H), 1.25 (br s, 3H); ${ }^{13} \mathrm{C} \mathrm{NMR} \mathrm{(75} \mathrm{MHz,} \mathrm{CDCl}_{3}$ ) $\delta$ 177.4, 169.3, 155.2, 144.5, 136.2, 134.2, 130.0, 128.8, 128.0, 127.7, 127.0, 126.9, 124.5, 80.0, 50.6, 48.3, 28.3, 18.1. Anal. Calcd for $\mathrm{C}_{22} \mathrm{H}_{25} \mathrm{~N}_{3} \mathrm{O}_{6}$ : C, 61.82; H, 5.90; N, 9.83. Found: C, 61.53; H, 6.06; N, 9.55.

Synthesis of 2-nitrobenzimidoyl chloride ${ }^{2}(10 \mathrm{e})$ and tert-butyl (S)-3-hydroxy-1-(2nitro- $N$-phenylbenzamido)-1-oxopropan-2-ylcarbamate (11e). 

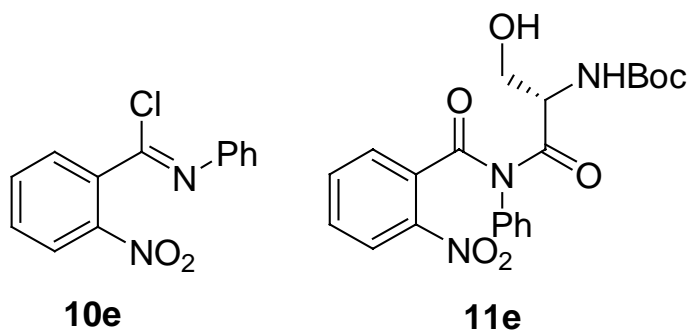

$N$-Benzyl-2-nitrobenzamide $(6.70 \mathrm{~g}, 27.7 \mathrm{mmol})$ was converted into crude $10 \mathrm{e}(6.55 \mathrm{~g}$, $91 \%$ ) as a yellow air-sensitive oil as described above for the preparation of 10a. A small sample of 10e was characterized: ${ }^{1} \mathrm{H} \mathrm{NMR}\left(300 \mathrm{MHz}, \mathrm{CDCl}_{3}\right) \delta 8.03(\mathrm{~d}, J=8.1 \mathrm{~Hz}, 1 \mathrm{H})$, $7.81-7.71(\mathrm{~m}, 2 \mathrm{H}), 7.63(\mathrm{td}, J=7.7,1.5 \mathrm{~Hz}, 1 \mathrm{H}), 7.43(\mathrm{t}, J=7.8 \mathrm{~Hz}, 2 \mathrm{H}), 7.24(\mathrm{t}, J=7.4$ $\mathrm{Hz}, 1 \mathrm{H}$, overlaps with $\mathrm{CHCl}_{3}$ signal), $7.09(\mathrm{~d}, J=7.7 \mathrm{~Hz}, 2 \mathrm{H})$. Crude 10e $(977 \mathrm{mg}, 3.74$ $\mathrm{mmol}$ ) was then reacted with Boc-L-serine (400 $\mathrm{mg}, 2.12 \mathrm{mmol}$ ) using the procedure for the preparation of 11a. Purification by flash chromatography on silica gel eluting with $60 \%$ ethyl acetate in hexanes afforded 11e (572 $\mathrm{mg}, 40 \%$ over two steps) as a yellow oil, which decomposes when stored at room temperature: ${ }^{1} \mathrm{H}$ NMR $\left(300 \mathrm{MHz}, \mathrm{CDCl}_{3}\right) \delta 8.12$ $(\mathrm{d}, J=8.3 \mathrm{~Hz}, 1 \mathrm{H}), 7.68(\mathrm{t}, J=7.6 \mathrm{~Hz}, 1 \mathrm{H}), 7.52-7.36(\mathrm{~m}, 7 \mathrm{H}), 5.41(\mathrm{br} \mathrm{d}, J=6.7 \mathrm{~Hz}$, $1 \mathrm{H}), 4.90(\mathrm{~m}, 1 \mathrm{H}), 3.99-3.92(\mathrm{~m}, 1 \mathrm{H}), 3.86-3.78(\mathrm{~m}, 1 \mathrm{H}), 2.02(\mathrm{~m}, 1 \mathrm{H}$, overlaps with EtOAc signal), 1.42 (s, 9H). HRMS Calcd for $\mathrm{C}_{21} \mathrm{H}_{23} \mathrm{~N}_{3} \mathrm{O}_{7}+\mathrm{H}$ : 430.1614. Found: 430.1633 .

\section{Synthesis of tert-Butyl (R)-1-(2-fluoro-6-nitro- $N$-phenylbenzamido)-1-oxobutan-2- ylcarbamate (11f).}<smiles>CCC(NC(C)(C)C)C(=O)N(C(=O)c1c(F)cccc1[N+](=O)[O-])c1ccccc1</smiles>

Thionyl chloride $(7.25 \mathrm{~mL}, 100 \mathrm{mmol})$ followed by DMF $(0.05 \mathrm{~mL})$ were added to $9 f$ $(5.20 \mathrm{~g}, 20.0 \mathrm{mmol})$. The reaction mixture was then heated to reflux for $1.5 \mathrm{~h}$. After this time the reaction was cooled to room temperature and concentrated under reduced pressure. Toluene $(3 \times 50 \mathrm{~mL})$ was added and removed three times under reduced pressure to give the crude imidoyl chloride 10f as a dark brown gum. Because of its instability, 10f was used directly in the next step without purification or characterization.

A solution of the $\mathbf{1 0 f}$ prepared above in $\mathrm{CH}_{2} \mathrm{Cl}_{2}(20 \mathrm{~mL})$ was added over $15 \mathrm{~min}$ to a solution of Boc-D- $\alpha$-Abu-OH $(4.47 \mathrm{~g}, 22.0 \mathrm{mmol})$ and triethylamine $(3.1 \mathrm{~mL}, 2.22 \mathrm{~g}$, $22.0 \mathrm{mmol})$ in $\mathrm{CH}_{2} \mathrm{Cl}_{2}(20 \mathrm{~mL})$ at $0{ }^{\circ} \mathrm{C}$. Once this addition was complete, the cooling bath was removed, and the reaction mixture was allowed to warm to room temperature. After $23 \mathrm{~h}$ at room temperature, additional Boc-D- $\alpha$-Abu-OH $(2.03 \mathrm{~g}, 10 \mathrm{mmol})$ and a solution of triethylamine $(1.4 \mathrm{~mL}, 1.01 \mathrm{~g}, 10.0 \mathrm{mmol})$ in $\mathrm{CH}_{2} \mathrm{Cl}_{2}(10 \mathrm{~mL})$ were added. After an additional $2 \mathrm{~h}$, the reaction mixture was filtered and the filtrate was washed with $0.1 \mathrm{M}$ aqueous hydrochloric acid $(3 \times 50 \mathrm{~mL})$, saturated aqueous $\mathrm{NaHCO}_{3}(3 \times 50 \mathrm{~mL})$ and water $(3 \times 50 \mathrm{~mL})$. The organic layer was then dried over $\mathrm{MgSO}_{4}$, filtered and the filtrate concentrated under reduced pressure. The resulting brown foam was suspended in toluene and the insoluble material was filtered off. The filtrate was purified by flash chromatography on silica gel, eluting first with toluene, then with $22 \%$ ethyl acetate in hexanes and finally with 50\% ethyl acetate in hexanes to give $\mathbf{1 1 f}(4.88 \mathrm{~g}, 55 \%)$ as a pale yellow solid: mp $118-120{ }^{\circ} \mathrm{C}$; the ${ }^{1} \mathrm{H}$ and ${ }^{13} \mathrm{C}$ NMR spectra indicated a mixture of 
rotamers; ${ }^{1} \mathrm{H}$ NMR $\left(400 \mathrm{MHz}, \mathrm{DMSO}-d_{6}\right) \delta 8.13(\mathrm{~d}, J=8.0 \mathrm{~Hz}, 1 \mathrm{H}), 7.85-7.80(\mathrm{~m}, 1 \mathrm{H})$, 7.76-7.71 (m, 1H), 7.56-7.37 (m, 5H), $7.21(\mathrm{~d}, J=7.4 \mathrm{~Hz}, 0.8 \mathrm{H}), 6.83$ (br s, 0.2H), 4.10 (br s, 1H), 1.70 (br s, 1H), 1.45 (br s, 1H), 1.39 (s, 1.8H), 1.35 (s, 7.2H), 0.72 (br s, 3H), ${ }^{13} \mathrm{C}$ NMR (100 MHz, DMSO- $\left.d_{6}\right) \delta 175.2,174.1,163.4,158.9,158.3,155.7,154.5,144.4$, 138.5, 136.3, 131.5, 129.7, 129.5, 128.9, 128.7, 124.2, 122.6 (d, $J=21.4$ Hz), 122.3, 120.5, 119.5, 78.9, 78.3, 77.9, 55.9, 28.2, 28.1, 24.2, 23.2, 10.6. Anal. Calcd for $\mathrm{C}_{22} \mathrm{H}_{24} \mathrm{FN}_{3} \mathrm{O}_{6}: \mathrm{C}, 59.32 ; \mathrm{H}, 5.43 ; \mathrm{N}, 9.43$. Found: C, 59.53; H, 5.29; N, 9.51.

\section{General procedure for ee determination for compounds 3a-3d and 3g-3h.}

Compound $3(0.2 \mathrm{mmol})$ was dissolved in a mixture of $\mathrm{CH}_{2} \mathrm{Cl}_{2}(0.5 \mathrm{~mL})$ and trifluoroacetic acid $(0.5 \mathrm{~mL})$. After stirring for $30 \mathrm{~min}$, the solvents were evaporated under reduced pressure at room temperature. (Note: Prolonged exposure of the quinazoline to strong acids causes partial racemization, and the time of contact with such acids should be minimized.) The residue was dissolved in $\mathrm{CH}_{2} \mathrm{Cl}_{2}(10 \mathrm{~mL})$, and the solution was extracted with $10 \%$ aqueous $\mathrm{K}_{2} \mathrm{CO}_{3}(10 \mathrm{~mL})$. The organic layer was then separated, dried over $\mathrm{Na}_{2} \mathrm{SO}_{4}$ and concentrated in vacuo. A 3-mg sample of the resulting amine was dissolved in $\mathrm{CHCl}_{3}(0.7 \mathrm{~mL})$, and $(R)-1$-(1-naphthyl)ethyl isocyanate $(3 \mu \mathrm{L})$ was added. Another 3-mg sample was similarly reacted with racemic 1-(1-naphthyl)ethyl isocyanate. After $18 \mathrm{~h}$ at room temperature the HPLC traces of both samples were compared. HPLC analyses were obtained using a Hypersil $5 \mu \mathrm{C} 18$ BDS column (250 x $4.6 \mathrm{~mm}$, Phenomenex), $1 \mathrm{~mL} / \mathrm{min}$ flow and UV detection at $254 \mathrm{~nm}$. Isocratic 60:40 acetonitrile/0.1\% TFA in water method was used for the samples derived from 3a,c and $\mathbf{d}$ and isocratic 50:50 acetonitrile/0.1\% TFA in water method was used for the samples derived from $\mathbf{3 b}, \mathbf{g}$ and $\mathbf{h}$. (Note: In the case of 3a, HPLC peak of the excess 1-(1naphthyl)ethyl isocyanate overlapped with the peaks of the reaction products.

Ethanolamine $(30 \mu \mathrm{L})$ was added in order to quench the isocyanate, and after $2 \mathrm{~h}$ the HPLC analysis was conducted again.)

\section{Synthesis of tert-butyl (S)-1-(5-methyl-4-oxo-3-o-tolyl-3,4-dihydroquinazolin-2- yl)ethylcarbamate (3a).}

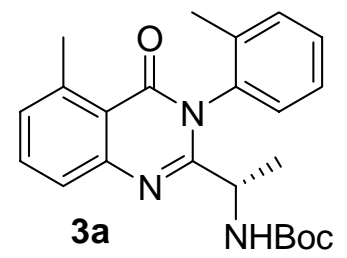

A flask with a suspension of zinc powder $(650 \mathrm{mg}, 10.0 \mathrm{mmol})$ in acetic acid $(4.0 \mathrm{~mL})$ was placed in a water bath $\left(20^{\circ} \mathrm{C}\right)$, and $3 \mathbf{a}(441 \mathrm{mg}, 1.00 \mathrm{mmol})$ was added. After the initial heat evolution, the reaction mixture was stirred at room temperature for $3.5 \mathrm{~h}$. After this time the mixture was filtered, and the filter cake was washed with acetic acid (2 $\times 2 \mathrm{~mL})$ followed by $\mathrm{CH}_{2} \mathrm{Cl}_{2}(2 \times 10 \mathrm{~mL})$. The filtrate was concentrated in vacuo, and the resulting residue was dissolved in $\mathrm{CH}_{2} \mathrm{Cl}_{2}(10 \mathrm{~mL})$. The solution was washed with saturated $\mathrm{NaHCO}_{3}(10 \mathrm{~mL})$, dried over $\mathrm{Na}_{2} \mathrm{SO}_{4}$ and concentrated in vacuo. The residue was purified by flash chromatography on silica gel eluting with $20 \%$ ethyl acetate in hexanes to afford 3a $(228 \mathrm{mg}, 58 \%)$ as a white solid: $\mathrm{mp} 130-137{ }^{\circ} \mathrm{C} ;[\alpha]_{\mathrm{D}}=-10.3^{\circ}(c$ $\left.0.14, \mathrm{MeOH}, 25{ }^{\circ} \mathrm{C}\right),>99 \%$ ee; according to the ${ }^{1} \mathrm{H}$ and ${ }^{13} \mathrm{C}$ NMR spectra, the obtained material is a mixture of two atropisomers in 60:40 ratio: ${ }^{1} \mathrm{H} \mathrm{NMR}\left(500 \mathrm{MHz}, \mathrm{CDCl}_{3}\right) \delta$ $7.61(\mathrm{td}, J=7.7,2.7 \mathrm{~Hz}, 1 \mathrm{H}), 7.57(\mathrm{~d}, J=8.1 \mathrm{~Hz}, 1 \mathrm{H}), 7.40(\mathrm{~m}, 3 \mathrm{H}), 7.29$ (br s, $0.4 \mathrm{H})$, $7.24(\mathrm{~d}, J=7.2 \mathrm{~Hz}, 1 \mathrm{H}), 7.16(\mathrm{~d}, J=8.5 \mathrm{~Hz}, 0.6 \mathrm{H}), 5.74(\mathrm{~m}, 1 \mathrm{H}), 4.57$ (br t, $J=6.7 \mathrm{~Hz}$, $0.4 \mathrm{H}), 4.32$ (quintet, $J=6.9 \mathrm{~Hz}, 0.6 \mathrm{H}), 2.83(\mathrm{~s}, 3 \mathrm{H}), 2.15(\mathrm{~s}, 1.2 \mathrm{H}), 2.13(\mathrm{~s}, 1.8 \mathrm{H}), 1.42$ 
$(\mathrm{s}, 9 \mathrm{H}), 1.30(\mathrm{~d}, J=6.6 \mathrm{~Hz}, 1.8 \mathrm{H}), 1.18(\mathrm{~d}, J=6.7 \mathrm{~Hz}, 1.2 \mathrm{H})$; NMR $\left(125 \mathrm{MHz}, \mathrm{CDCl}_{3}\right) \delta$ 162.2, 161.9, 157.6, 157.2, 154.7, 154.6, 148.9, 148.8, 141.8, 136.2, 136.1, 133.7, 132.0, $131.4,129.8,129.65,129.61,128.7,128.6,127.8,127.2,125.0,119.6,119.4,79.6,47.6$, 28.41, 28.37, 23.06, 23.04, 21.4, 20.0, 17.8, 17.5. Anal. Calcd for $\mathrm{C}_{23} \mathrm{H}_{27} \mathrm{~N}_{3} \mathrm{O}_{3}: \mathrm{C}, 70.21$; H, 6.92; N, 10.68. Found: C, 70.19; H, 6.92; N, 10.68.

Synthesis of tert-butyl (S)-1-(5-methyl-4-oxo-3-phenyl-3,4-dihydroquinazolin-2yl)ethylcarbamate $(3 b)$.

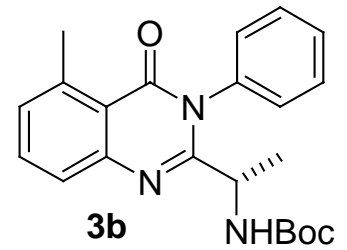

To zinc powder $(3.82 \mathrm{~g}, 58.8 \mathrm{mmol})$ suspended in acetic acid $(10 \mathrm{~mL})$ a solution of $\mathbf{1 1 b}$ $(1.50 \mathrm{~g}, 3.92 \mathrm{mmol})$ in acetic acid was added portionwise over $1 \mathrm{~h}$. After $2 \mathrm{~h}$ at room temperature, more zinc powder $(1.27 \mathrm{~g}, 19.5 \mathrm{mmol})$ was added, and the reaction was stirred for a further $1 \mathrm{~h}$. The procedure for the preparation of $\mathbf{3 a}$ was then followed. Final purification by flash chromatography on silica gel eluting with a gradient from $100 \%$ hexanes to $40 \%$ ethyl acetate in hexanes, afforded $\mathbf{3 b}(900 \mathrm{mg}, 60 \%)$ as a white solid: $\mathrm{mp}$ $164-166{ }^{\circ} \mathrm{C} ;[\alpha]_{\mathrm{D}}=-4.63^{\circ}\left(c 0.2, \mathrm{MeOH}, 25^{\circ} \mathrm{C}\right), 98 \%$ ee; ${ }^{1} \mathrm{H}$ NMR $\left(300 \mathrm{MHz}, \mathrm{CDCl}_{3}\right) \delta$ 7.64-7.48 (m, 5H), $7.36(\mathrm{~d}, J=7.4 \mathrm{~Hz}, 1 \mathrm{H}), 7.29-7.19\left(\mathrm{~m}, 2 \mathrm{H}\right.$, overlaps with $\mathrm{CHCl}_{3}$ signal), 5.68 (br s, 1H), $4.50(\mathrm{~m}, 1 \mathrm{H}), 2.82(\mathrm{~s}, 3 \mathrm{H}), 1.42(\mathrm{~s}, 9 \mathrm{H}), 1.26(\mathrm{~d}, J=6.6 \mathrm{~Hz}, 3 \mathrm{H})$; ${ }^{13} \mathrm{C}$ NMR $\left(75 \mathrm{MHz}, \mathrm{CDCl}_{3}\right) \delta 163.2,149.2,142.1,136.7,134.1,130.8,130.0,129.8$, 129.4, 129.0, 125.8, 119.9, 80.0, 48.1, 28.8, 23.4, 21.2; Anal. Calcd for $\mathrm{C}_{22} \mathrm{H}_{25} \mathrm{~N}_{3} \mathrm{O}_{3}$ : C, 69.64; H, 6.64; N, 11.07. Found: C, 69.43; H, 6.65; N, 10.82 .

\section{Synthesis of tert-butyl (S) 1-(3-(3,5-difluorophenyl)-5-methyl-4-oxo-3,4-} dihydroquinazolin-2-yl)ethylcarbamate (3c).<smiles>Cc1cccc2nc([C@H](C)NC(=O)OC(C)(C)C)n(-c3cc(F)cc(F)c3)c(=O)c12</smiles>

To a solution of 11c $(1.00 \mathrm{~g}, 2.16 \mathrm{mmol})$ in acetic acid $(30 \mathrm{~mL})$, zinc powder $(3.40 \mathrm{~g}$, $52.3 \mathrm{mmol}$ ) was added. After stirring for $13 \mathrm{~h}$, the procedure for the preparation of $\mathbf{3 a}$ was followed. Final purification by flash chromatography on silica gel, eluting with $25 \%$ ethyl acetate in hexanes, afforded 3c (357 mg, 40\%) as a white solid: $\mathrm{mp} 151-153{ }^{\circ} \mathrm{C}$; $[\alpha]_{\mathrm{D}}=+7.2^{\circ}\left(c 0.13, \mathrm{MeOH}, 25{ }^{\circ} \mathrm{C}\right), 94 \%$ ee; ${ }^{1} \mathrm{H} \mathrm{NMR}\left(300 \mathrm{MHz}, \mathrm{CDCl}_{3}\right) \delta 7.63(\mathrm{t}, J=$ $7.7 \mathrm{~Hz}, 1 \mathrm{H}), 7.55(\mathrm{~d}, J=7.3 \mathrm{~Hz}, 1 \mathrm{H}), 7.26\left(\mathrm{~d}, J=6.9 \mathrm{~Hz}, 1 \mathrm{H}\right.$, overlaps with $\mathrm{CHCl}_{3}$ signal), $7.00(\mathrm{~m}, 2 \mathrm{H}), 6.87(\mathrm{~d}, J=7.9 \mathrm{~Hz}), 5.51(\mathrm{br} \mathrm{d}, J=7.2 \mathrm{~Hz}, 1 \mathrm{H}), 4.47$ (quintet, $J=$ $7.0 \mathrm{~Hz}, 1 \mathrm{H}), 2.81(\mathrm{~s}, 3 \mathrm{H}), 1.41(\mathrm{~s}, 9 \mathrm{H}), 1.33(\mathrm{~d}, J=6.7 \mathrm{~Hz}, 3 \mathrm{H}) ;{ }^{13} \mathrm{C} \mathrm{NMR}(75 \mathrm{MHz}$, $\left.\mathrm{CDCl}_{3}\right) \delta 163.6(\mathrm{~J}=251.7,13.3 \mathrm{~Hz}), 163.2(\mathrm{~J}=251.7,13.3 \mathrm{~Hz}), 162.4,156.4,154.8$, $148.5,141.7,138.5(\mathrm{t}, J=12.2 \mathrm{~Hz}), 134.08,130.01,125.6,119.2,113.0(\mathrm{~d}, J=23.5 \mathrm{~Hz})$, $105.6(\mathrm{t}, J=25.1 \mathrm{~Hz}), 79.9,28.3,23.0,20.7$; Anal. Calcd for $\mathrm{C}_{22} \mathrm{H}_{23} \mathrm{~F}_{2} \mathrm{~N}_{3} \mathrm{O}_{3}: \mathrm{C}, 63.60 ; \mathrm{H}$, $5.58 ; \mathrm{N}, 10.12$. Found: C, 63.82; H, 5.61; N, 9.98.

Synthesis of tert-butyl (S)-1-(3-benzyl-4-oxo-3,4-dihydroquinazolin-2yl)ethylcarbamate (3d). 


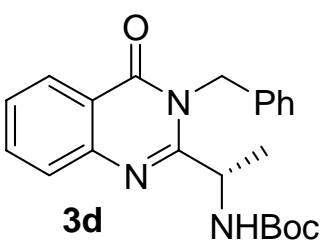

11d (300 mg, $0.703 \mathrm{mmol})$ was reduced by zinc powder (450 mg, $7.03 \mathrm{mmol})$ in acetic acid $(4 \mathrm{~mL})$ using the procedure for the preparation of 3a. Final purification by flash chromatography on silica gel eluting with $25 \%$ ethyl acetate in hexanes afforded $\mathbf{3 d}$ (188 mg, 71\%) as a white solid: $\mathrm{mp} 123-125^{\circ} \mathrm{C} ;[\alpha]_{\mathrm{D}}=-179.0^{\circ}\left(c 0.11, \mathrm{MeOH}, 25^{\circ} \mathrm{C}\right),>99 \%$ ee; ${ }^{1} \mathrm{H}$ NMR $\left(500 \mathrm{MHz}, \mathrm{CDCl}_{3}\right) \delta 8.33(\mathrm{dd}, J=7.9,1.1 \mathrm{~Hz}, 1 \mathrm{H}), 7.76(\mathrm{td}, J=7.6,1.2 \mathrm{~Hz}$, $1 \mathrm{H}), 7.66(\mathrm{~d}, J=8.1 \mathrm{~Hz}, 1 \mathrm{H}), 7.49(\mathrm{t}, J=7.5 \mathrm{~Hz}, 1 \mathrm{H}), 7.32-7.26(\mathrm{~m}, 5 \mathrm{H}$, overlaps with $\mathrm{CHCl}_{3}$ signal), $5.74(\mathrm{~d}, J=15.9 \mathrm{~Hz}, 1 \mathrm{H}), 5.58(\mathrm{~d}, J=8.2 \mathrm{~Hz}, 1 \mathrm{H}), 5.33(\mathrm{~d}, J=15.8 \mathrm{~Hz}$, $1 \mathrm{H}), 5.04$ (quintet, $J=7.2 \mathrm{~Hz}, 1 \mathrm{H}), 1.43(\mathrm{~s}, 9 \mathrm{H}), 1.22(\mathrm{~d}, J=6.3 \mathrm{~Hz}, 3 \mathrm{H}) ;{ }^{13} \mathrm{C} \mathrm{NMR}(75$ $\left.\mathrm{MHz}, \mathrm{CDCl}_{3}\right) \delta 162.4,158.2,154.9,147.0,136.2,134.4,128.9,127.8,127.2,127.1$, 127.0, 120.7, 79.9, 47.5, 46.2, 28.4, 21.1. Anal. Calcd for $\mathrm{C}_{22} \mathrm{H}_{25} \mathrm{~N}_{3} \mathrm{O}_{3}$ : C, 69.64; $\mathrm{H}, 6.64$; N, 11.07. Found: C, 69.39; H, 6.72; N, 10.85.

\section{Synthesis of tert-butyl $(R)$-2-hydroxy-1-(4-oxo-3-phenyl-3,4-dihydroquinazolin-2- yl)ethylcarbamate (3e).}<smiles>O=C(O)[C@H](CO)c1nc2ccccc2c(=O)n1-c1ccccc1</smiles>

Zinc powder (165 mg, $2.53 \mathrm{mmol}$ ) was added to a solution of 11e (286 mg, $0.667 \mathrm{mmol})$ in acetic acid $(3.5 \mathrm{~mL})$, and the mixture was stirred for $6 \mathrm{~h}$. The procedure for the preparation of 3a was then followed. Final purification by flash chromatography on silica gel, eluting with 50\% ethyl acetate in hexanes, afforded $\mathbf{3 e}(145 \mathrm{mg}, 57 \%)$ as a white solid: $\mathrm{mp} 80-83{ }^{\circ} \mathrm{C},[\alpha]_{\mathrm{D}}=-6.7^{\circ}\left(c 0.06, \mathrm{MeOH}, 25^{\circ} \mathrm{C}\right), 94 \%$ ee; ${ }^{1} \mathrm{H}$ NMR $(500$ $\left.\mathrm{MHz}, \mathrm{CDCl}_{3}\right) \delta 8.29(\mathrm{dd}, J=7.9,1.5 \mathrm{~Hz}, 1 \mathrm{H}), 7.79(\mathrm{t}, J=8.1 \mathrm{~Hz}, 1 \mathrm{H}), 7.71(\mathrm{~d}, J=8.2$ $\mathrm{Hz}, 1 \mathrm{H}), 7.59-7.51(\mathrm{~m}, 4 \mathrm{H}), 7.35(\mathrm{dd}, J=7.8,2.2 \mathrm{~Hz}, 1 \mathrm{H}), 7.27$ (m, 1H, overlaps with $\mathrm{CHCl}_{3}$ signal), 5.58 (br s, $\left.1 \mathrm{H}\right), 4.59$ (br s, $\left.1 \mathrm{H}\right), 3.86(\mathrm{~d}, J=11.2 \mathrm{~Hz}, 1 \mathrm{H}), 3.75(\mathrm{~m}, 1 \mathrm{H})$, 3.47 (br s, $1 \mathrm{H}), 1.32$ (s, 9H); ${ }^{13} \mathrm{C} \mathrm{NMR}\left(125 \mathrm{MHz}, \mathrm{CDCl}_{3}\right) \delta 162.0,155.2,154.8,146.3$, 135.8, 134.8, 130.2, 129.9, 129.7, 129.1, 128.8, 128.3, 127.6, 127.3, 127.2, 121.3, 120.0, 80.2, 64.2, 52.3, 28.3. HRMS Calcd for $\mathrm{C}_{21} \mathrm{H}_{25} \mathrm{~N}_{3} \mathrm{O}_{4}+\mathrm{H}: 382.1767$. Found: 382.1778.

The ee of compound 3e was determined as follows. A 4-mg sample of $\mathbf{3 e}$ was reacted with $(S)$-2-methoxy-2-phenylacetyl chloride $(10 \mu \mathrm{L})$ in $\mathrm{CH}_{2} \mathrm{Cl}_{2}(0.3 \mathrm{~mL}) /$ pyridine $(0.1$ $\mathrm{mL})$. Another 4-mg sample was similarly reacted with racemic 2-methoxy-2phenylacetyl chloride. After $5 \mathrm{~h}$ at room temperature, the HPLC traces of both samples were compared. HPLC analyses were obtained using a Hypersil $5 \mu \mathrm{C} 18$ BDS column ( $250 \times 4.6 \mathrm{~mm}$, Phenomenex), $1 \mathrm{~mL} / \mathrm{min}$ flow and UV detection at $254 \mathrm{~nm}$. Isocratic 50:50 acetonitrile/0.1\% TFA in water method was used.

Synthesis of tert-butyl (R)-1-(5-fluoro-4-oxo-3-phenyl-3,4-dihydroquinazolin-2yl)propylcarbamate (3f). 
<smiles>CCC(NC(C)(C)C)c1nc2cccc(F)c2c(=O)n1-c1ccccc1</smiles>

$11 f(445 \mathrm{mg}, 1.00 \mathrm{mmol}$ ) was dissolved in acetic acid (5 mL). Zinc dust (393 mg, 6.00 mmol) was added in three portions with 5 min intervals. Each addition was associated with a slight temperature increase (ranging from 3 to $6^{\circ} \mathrm{C}$ ). After $17.5 \mathrm{~h}$ the reaction mixture was filtered, and the filtrate was concentrated under reduced pressure. The resulting residue was dissolved in ethyl acetate $(15 \mathrm{~mL})$ and washed with saturated aqueous $\mathrm{NaHCO}_{3}(3 \times 15 \mathrm{~mL})$, water $(2 \times 15 \mathrm{~mL})$ and brine $(15 \mathrm{~mL})$. The organic solution was dried over $\mathrm{MgSO}_{4}$, filtered and the filtrate concentrated under reduced pressure. The resulting light brown foam was purified by flash chromatography on silica gel eluting with $17 \%$ ethyl acetate in hexanes to afford $\mathbf{3 f}(267 \mathrm{mg}, 67 \%)$ as a colorless solid: mp 114-116 ${ }^{\circ} \mathrm{C}$; $97 \%$ ee; the ${ }^{1} \mathrm{H}$ NMR spectrum indicated the presence of two rotational isomers ( $~ 9: 1$ ratio), which coalesce when the spectrum is acquired at $50{ }^{\circ} \mathrm{C}$; ${ }^{1} \mathrm{H}$ NMR (400 MHz, DMSO- $\left.d_{6}\right) \delta 7.83(\mathrm{dt}, J=8.2,5.8 \mathrm{~Hz}, 1 \mathrm{H}), 7.62-7.49(\mathrm{~m}, 5 \mathrm{H}), 7.39$ (br d, $J=8.2 \mathrm{~Hz}, 1 \mathrm{H}), 7.30(\mathrm{dd}, J=10.7,8.2 \mathrm{~Hz}, 1 \mathrm{H}), 7.22(\mathrm{~d}, J=7.7 \mathrm{~Hz}, 0.9 \mathrm{H}), 6.71$ (br s, 0.1H), 3.97-3.92 (m, 1H), 1.76-1.68 (m, 1H), 1.59-1.48 (m, 1H), 1.33 (br s, 8.1H), 1.23 (br s, 0.9H), $0.63(\mathrm{t}, J=7.2 \mathrm{~Hz}, 3 \mathrm{H}) ;{ }^{13} \mathrm{C}$ NMR $\left(100 \mathrm{MHz}, \mathrm{DMSO}-d_{6}\right) \delta 160.5(\mathrm{~d}, J=$ $264.0 \mathrm{~Hz}), 159.1,158.3(\mathrm{~d}, J=3.6 \mathrm{~Hz}), 155.5,149.1,136.0,135.4(\mathrm{~d}, J=11.4 \mathrm{~Hz})$, 129.6, 129.3, 129.1 (br s), 128.7, 123.0 (d, $J=4.0 \mathrm{~Hz}$ ), 113.2, (d, $J=20.6 \mathrm{~Hz}$ ), 110.2 (d, $J=5.3 \mathrm{~Hz}$ ), 78.1, 54.1, 28.2, 25.7, 10.8. Anal. Calcd for $\mathrm{C}_{22} \mathrm{H}_{24} \mathrm{FN}_{3} \mathrm{O}_{3}: \mathrm{C}, 66.48 ; \mathrm{H}, 6.09$; N, 10.57. Found: C, 66.30; H, 5.96; N, 10.47.

The ee of compound $\mathbf{3 f}$ was determined as follows. A sample of $\mathbf{3 f}$ was analyzed by chiral HPLC and compared with a mixture of the R \& S enantiomers which were analyzed under identical conditions. Chiralpak AD, 4.6 x $150 \mathrm{~mm}, 20^{\circ} \mathrm{C}, 90: 10$ hexanes:isopropanol, $1 \mathrm{~mL} / \mathrm{min}$. The ee was calculated from the area under the respective peaks@230 nm and was determined to be $>98 \%$.

\section{Synthesis of tert-butyl (S)-1-(3-(2,6-difluorophenyl)-4-oxo-3,4-dihydroquinazolin-2- yl)ethylcarbamate (3g).}<smiles>C[C@H](NC(=O)OCc1ccccc1)c1nc2ccccc2c(=O)n1-c1c(F)cccc1F</smiles>

To a suspension of $9 \mathrm{~g}(30.0 \mathrm{~g}, 108 \mathrm{mmol})$ in THF $(300 \mathrm{~mL})$ at $0{ }^{\circ} \mathrm{C}$ was added KHMDS $(0.5 \mathrm{M}$ solution in toluene, $216 \mathrm{~mL}, 108 \mathrm{mmol})$. The reaction was then removed from the cooling bath and stirred for $10 \mathrm{~min}$, after which time Boc-L-Ala-OSu (31.0 g, $108 \mathrm{mmol})$ was added. After $1 \mathrm{~h}$ at ambient temperature, the reaction was concentrated in vacuo, and the residue was dissolved in ethyl acetate $(600 \mathrm{~mL})$. The solution was then washed with water $(3 \times 200 \mathrm{~mL})$, aqueous $0.5 \mathrm{M}$ hydrochloric acid $(200 \mathrm{~mL})$ and brine $(100 \mathrm{~mL})$. The organic layer was concentrated in vacuo to give imide $\mathbf{1 1 g}$ as a yellow foam, which was dissolved directly in acetic acid $(600 \mathrm{~mL})$. Zinc dust $(42.3 \mathrm{~g}, 647 \mathrm{mmol})$ was added to this solution in three portions over $2 \mathrm{~h}$. After this addition was complete, the reaction was stirred at ambient temperature overnight. The procedure for the preparation of 3a was then followed. Final purification by flash chromatography on silica gel eluting with 
$25 \%$ ethyl acetate in hexanes afforded $3 \mathrm{~g}$ (21.2 $\mathrm{g}, 49 \%$ over two steps) as a white foam: mp 108-110 ${ }^{\circ} \mathrm{C}$; $93 \%$ ee; ${ }^{1} \mathrm{H}$ NMR $\left(300 \mathrm{MHz}, \mathrm{DMSO}-d_{6}\right) \delta 8.17(\mathrm{~d}, J=7.6 \mathrm{~Hz}, 1 \mathrm{H}), 7.95$ $(\mathrm{t}, J=7.2 \mathrm{~Hz}, 1 \mathrm{H}), 7.79(\mathrm{~d}, J=8.1 \mathrm{~Hz}, 1 \mathrm{H}), 7.73-7.63(\mathrm{~m}, 1 \mathrm{H}), 7.64(\mathrm{t}, J=7.3 \mathrm{~Hz}, 1 \mathrm{H})$, 7.47-7.34 (m, 3H), 4.38 (quintet, $J=7.3 \mathrm{~Hz}, 1 \mathrm{H}), 1.32$ (d, $J=6.9 \mathrm{~Hz}, 3 \mathrm{H}), 1.30(\mathrm{~s}, 9 \mathrm{H})$; ${ }^{13} \mathrm{C}$ NMR (75 MHz, DMSO-d6) $\delta 160.3,158.1(\mathrm{~d}, J=256.6 \mathrm{~Hz}), 157.5(\mathrm{~d}, J=248.7 \mathrm{~Hz})$, $156.2,154.4,146.5,135.6,132.3(\mathrm{t}, J=10.3 \mathrm{~Hz}), 127.8,127.5,126.6,119.7,113.2-112.3$ (m), 78.1, 48.0, 28.1, 18.6. Anal. Calcd for $\mathrm{C}_{21} \mathrm{H}_{21} \mathrm{~F}_{2} \mathrm{~N}_{3} \mathrm{O}_{3}$ 0.15 EtOAc: C, 62.57; H, 5.40; N, 10.13; F, 9.16. Found: C, 62.28; H, 5.19; N, 10.30; F, 9.23.

\section{Synthesis of tert-butyl (S)-1-(3-(2,6-difluorophenyl)-5-methyl-4-oxo-3,4- dihydroquinazolin-2-yl)ethylcarbamate $(3 \mathrm{~h})$.}<smiles>Cc1cccc2nc([C@H](C)NC(=O)OCc3ccccc3)n(-c3c(F)cccc3F)c(=O)c12</smiles>

Solid KHMDS (10.6 g, $53.0 \mathrm{mmol})$ was added to a solution of $9 \mathbf{h}(15.5 \mathrm{~g}, 53.0 \mathrm{mmol})$ in THF $(200 \mathrm{~mL})$ at $0{ }^{\circ} \mathrm{C}$. The reaction mixture was stirred for $10 \mathrm{~min}$ at $0{ }^{\circ} \mathrm{C}$, after which time Boc-L-Ala-OSu $(15.2 \mathrm{~g}, 53.0 \mathrm{mmol})$ was added. After stirring for an additional 15 min at the same temperature, the reaction mixture was concentrated, and the residue was dissolved in ethyl acetate $(300 \mathrm{~mL})$ and washed with water $(3 \times 100 \mathrm{~mL})$. The organic layer was dried over $\mathrm{MgSO}_{4}$, filtered and concentrated in vacuo to afford the crude imide, which was used without purification in the subsequent step. The crude material was dissolved in acetic acid $(65 \mathrm{~mL})$ and treated with zinc powder $(11.2 \mathrm{~g}, 172 \mathrm{mmol})$ in three portions. After stirring for $2 \mathrm{~h}$, the procedure for the preparation of 3a was followed.

Final purification by flash chromatography on silica gel, eluting with $30 \%$ ethyl acetate in hexanes, afforded $\mathbf{3 h}(4.35 \mathrm{~g}, 20 \%)$ as a white solid: $\mathrm{mp} 123-125{ }^{\circ} \mathrm{C} ;{ }^{1} \mathrm{H}$ NMR $(400 \mathrm{MHz}$, $\left.\mathrm{CDCl}_{3}\right) \delta 7.65-7.46(\mathrm{~m}, 3 \mathrm{H}), 7.28(\mathrm{br} \mathrm{s}, 1 \mathrm{H}), 7.28-7.25(\mathrm{~m}, 1 \mathrm{H}$, partially overlaps with $\mathrm{CHCl}_{3}$ signal), 7.16-7.11 (m, 2H), 5.80 (br d, $\left.J=8.4 \mathrm{~Hz}, 1 \mathrm{H}\right), 4.49$ (m, 1H), $2.82(\mathrm{~s}, 3 \mathrm{H})$, 1.42 (s, 9H), 1.33 (d, $J=6.4 \mathrm{~Hz}, 3 \mathrm{H}) ;{ }^{13} \mathrm{C}$ NMR (100 MHz, DMSO-d $\left.{ }_{6}\right) \delta 163.8,161.3$, $158.9(\mathrm{dd}, J=252.6,3.8 \mathrm{~Hz}), 158.5$ (dd, $J=248.7,3.8 \mathrm{~Hz}), 156.8,155.2,148.8,141.4$, $135.1,132.8(\mathrm{t}, J=10.0 \mathrm{~Hz}), 130.6,126.3,118.9,113.8(\mathrm{t}, J=17.2 \mathrm{~Hz}), 114.1-113.0$ (m), 78.8, 55.5, 48.6, 28.8, 23.1, 19.3. Anal. Calcd for $\mathrm{C}_{22} \mathrm{H}_{23} \mathrm{~F}_{2} \mathrm{~N}_{3} \mathrm{O}_{3}$ : C, 63.60; $\mathrm{H}, 5.58$; N, 10.12. Found: C, 63.23; H, 5.60; N, 9.99.

\section{References}

${ }^{1}$ Agwada, V. C. J. Chem. Eng. Data 1984, 29, 231.

${ }^{2}$ Gruszecki, W.; Gruszecka, M.; Bradaczek, H. Lieb. Ann. Chem. 1988, 331. 